\title{
Reglamento interno para el cumplimiento de los derechos de las adolescentes
}

\author{
Internal regulation for the fulfillment of the rights of adolescents
}

Regulamento interno para o cumprimento dos direitos do adolescente

Artículo recibido en abril 2019

Arbitrado en mayo 2019

Publicado en julio 2019

\section{Analuz de Jesús Garnica Zubieta}

a.garnica.z@gmail.com
RESUMEN

ABSTRACT

RESUMO
Los centros de acogidas en Bolivia, presentan un alto índice de incumplimiento de los derechos hacia las adolescentes, en tal sentido la investigación tuvo como objetivo proponer un reglamento interno para el cumplimiento de los derechos de las adolescentes. La investigación fue de tipo descriptivo, el diseño fue documental de campo, los métodos empleados fueron análisis, histórico, otros. La técnica e instrumentos fueron la encuesta, entrevista y el instrumento fue el cuestionario. La población usada fue la perteneciente en el centro de acogida. La muestra se conformó por 24 adolescentes, 6 encargados del personal del centro de acogida y las entrevistas fueron a 1 Juez del Juzgado Público de la Niñez y Adolescencia, 2 abogados de la Defensoría de la Niñez y Adolescencia. Como resultado se obtuvo la elaboración del reglamento interno para ser aplicado en el centro, de esta manera garantizar en las adolescentes el cumplimiento de sus derechos.

Palabras clave: Derechos de los niños, adolescentes; reglamento, derechos humanos

The shelters in Bolivia present a high rate of non-compliance with the rights of adolescents, in this sense the research aimed to propose an internal regulation for the fulfillment of the rights of adolescents. The research was descriptive, the design was field documentary, the methods used were analysis, historical, and others. The technique and instruments were the survey, interview and the instrument were the questionnaire. The population used was the one belonging to the reception center. The sample consisted of 24 adolescents, 6 staff in charge of the reception center and the interviews were conducted with 1 Judge from the Public Court for Children and Adolescents, 2 lawyers from the Ombudsman's Office for Children and Adolescents. As a result, the elaboration of the internal regulations was obtained to be applied in the center, in this way guaranteeing adolescents the fulfillment of their rights.

Key words: Rights of children and adolescents; adolescents; reception center; regulations, human rights

Os abrigos na Bolívia apresentam um alto índice de descumprimento dos direitos dos adolescentes, nesse sentido a pesquisa teve como objetivo propor um regulamento interno para o cumprimento dos direitos dos adolescentes. A pesquisa foi descritiva, o desenho foi documental de campo, os métodos utilizados foram análise, histórico, entre outros. A técnica e os instrumentos foram o inquérito, a entrevista e o instrumento foi o questionário. A população utilizada foi a pertencente ao centro de acolhimento. A amostra foi composta por 24 adolescentes, 6 funcionários responsáveis pela unidade de acolhimento e as entrevistas foram realizadas com 1 Juiz da Vara Pública da Criança e do Adolescente, 2 advogados da Ouvidoria da Criança e do Adolescente. Com isso, obteve-se a elaboração do regimento interno para aplicação no centro, garantindo assim aos adolescentes o cumprimento de seus direitos. Direitos da criança e do adolescente; adolescentes; centro de acolhimento; regulamentos, direitos humanos

Palavras-chave: Corrupção; observatório anticorrupção; direito Comparado, direito penal, Bolívia 


\section{INTRODUCCIÓN}

Las adolescentes son las personas más afectadas por las diferencias y problemas que hay en el mundo, en especial en los centros de acogida; por esto, las diferentes organizaciones mundiales que velan por la protección de los derechos de la niñez y adolescencia regulan las relaciones internacionales y han sentido la necesidad de crear organismos que se ocupen especialmente de los niños y adolescentes para poder brindarles una mejor atención y protección.

Por ello, frente al incumplimiento de los derechos en los que se involucran a las adolescentes de los centros de acogida, se requiere de las instituciones convocadas a desarrollar modalidades de intervención que promuevan la atención personalizada, el respeto de los aspectos socioculturales, su inclusión en espacios educativos, de salud, recreación y capacitación inherentes al proceso evolutivo de cada adolescente.

Así que, la supervisión de estas instituciones tiene que tener presente la protección integral, interés superior y efectividad de los derechos; todas las instituciones deben garantizar a las adolescentes la protección y cuidados que sean necesarios teniendo en cuenta el interés superior de las adolescentes.

De esta manera, al buscar el bienestar de las adolescentes se debe incrementar el presupuesto en protección social, retomar el modelo de los Programas de Atención a la Adolescencia, complementarlos con la incorporación del trabajo con familias o cuidadores, coordinar con instancias departamentales, municipales y organizaciones no gubernamentales para la ampliación de la cobertura de atención, serian medidas que contribuirían a garantizar los derechos de las adolescentes, pero sobre todo el derecho a la protección y cuidado. De esta manera tienen la obligación y las condiciones necesarias para viabilizar esta recomendación, sin embargo, el trabajo necesariamente implica la coordinación con otras instancias del estado como el Ministerio de Educación y Salud.

Bolivia con el fin de preservar las generaciones futuras, garantizando la dignidad y el valor de las personas, la igualdad de los derechos del hombre, mantener la justicia y el respeto a las obligaciones emanadas de los tratados y otras fuentes del derecho internacional, ha unido sus esfuerzos para que el respectivo los Gobiernos Autónomos Municipales establezca una organización municipal y nacional que se encargue del control riguroso de los centros de acogida.

En efecto, es importante tomar en cuenta para el estudio, el desarrollo jurisprudencial de la Corte Interamericana de Derechos Humanos en relación con los derechos de las adolescentes, y la participación en el centro de acogida 10 de Noviembre por la situación del incumplimiento de los derechos humanos que está presente en Bolivia. 
Aunado a ello, es importante que en el lineamiento de protección de los derechos del adolescentes, se deba velar para que los centros de acogida estén capacitados para generar protección y cumplir con el rol de resguardo para ellos; de lo contrario se debe revertir definitivamente la situación haciéndose cargo de estas adolescentes por otros medios; como educacional, ya que debe generarse un cambio radical en la educación que garantice y beneficie a todos las adolescentes del centro de acogida 10 de noviembre sin importar la condición social, económica o cultural, la igualdad de oportunidades y por una excelente calidad educacional.

Seguidamente el contexto educacional también es clave para la concientización de los derechos de las adolescentes, los profesionales a cargo deben estar capacitados para resguardar a las adolescentes, es así como los responsables del centro se hacen participes inminentemente de los procesos de desarrollo y socialización que van generando las adolescentes a partir del inicio de su etapa de educación secundaria.

En un contexto educativo delicado, las adolescentes que padecen problemáticas graves derivan sus emociones y sentimientos hacia estos sujetos que deben velar por educarlos, pero que de algún modo se vinculan sentimentalmente con ellos, es por eso que necesario una reglamentación interna en dicho centro para que éste tenga un mejor funcionamiento.

Por tal motivo el objeto de estudio de la investigación se basa en los derechos de Las adolescentes en la Constitución Política del Estado y el Código Niña, Niño y Adolescente. De tal manera se plantea como objeto principal de la investigación, proponer un Reglamento Interno para el cumplimiento de los derechos de las adolescentes del Centro de Acogida 10 de noviembre en la ciudad de Potosí- Bolivia.

De esta manera, al tener políticas de protección hacia las adolescentes, se busca tener la construcción de una cultura que combata la violencia y la discriminación, además ayuda a promover la tolerancia, la democracia y la paz. Por otro lado, reduce la descentralización en búsqueda de la equidad creando políticas sociales con medidas especiales de protección para que las adolescentes puedan llevar una vida sin incumplimiento de sus derechos por ende se necesita incorporar una reglamentación interna al centro de acogida.

\section{Marco histórico}

Cárdenas (2012) en el trabajo realizado sobre la percepción de los trabajadores sociales, educadores y psicólogos sobre los centros de acogida para menores y apoyado fundamentalmente sobre la teoría del aprendizaje social de Bandura, establece que en caso de los menores en los centros de acogida aún se tienen incumplimientos de los derechos en los niveles psicológicos y físicos fundamentalmente. 
En este mismo orden de ideas, del incumplimiento de los derechos de las adolescentes en los centros de acogida Alcántara, (2010), plantea que el incumplimiento de los Derechos de las adolescentes tiene una afectación desde dos ámbitos, la propia familia y los educadores, profesionales que trabajan en estos centros de acogida. Señalando que el incumplimiento de los derechos de las adolescentes, quedan manifestadas visiblemente entre el maltrato psicológico y físico.

\section{Generalidades sobre el cumplimiento de los derechos de las adolescentes en el centro de acogida 10 de noviembre y sus alcances en el ámbito de sus derechos}

El incumplimiento de derechos de las adolescentes del centro es entendido como cualquier falta de ejecución, acto violento hacia ellas, podría resultar, en daño físico, sexual o psicológico, incluyendo las amenazas de realizar tal acto, coacción o la privación arbitraria de libertad, produciéndose estos en la vida pública o privada, es una de las formas de violencia que más atención social e institucional se ha recibido en Bolivia y especialmente en el departamento de Potosí, debido al impacto que ha producido en la sociedad.

\section{Derechos de las adolescentes}

Los derechos humanos son garantías universales que protegen a los individuos y grupos frente a acciones $u$ omisiones que puedan afectar sus libertades y su dignidad humana.

La Declaración Universal de los Derechos Humanos fue aprobada por la asamblea general de las naciones unidas el 10 De diciembre de 1948, considerando en ella que la paz, la justicia y la libertad en el mundo tiene como base el reconocimiento de la dignidad intrínseca y de los derechos iguales e inalienables de todos los miembros de la familia humana, consiguiendo para ellos implementar las relaciones amistosas entre los miembros que la conforman.

\section{Declaración de los Derechos de la Niñez y Adolescencia}

Todos y cada uno de los derechos de la infancia son inalienables e irrenunciables, por lo que ninguna persona puede vulnerarlos o desconocerlos bajo ninguna circunstancia. Varios documentos consagran los derechos de la infancia a nivel internacional, entre ellos la declaración de los derechos del niño y así mismo de Las adolescentes (Morlachetti, 2014), este documento reconoce a los niños y adolescentes como sujetos de derecho, pero convierten a los Estados y a los adultos en titulares de la obligación de respetarlos y hacerlos respetar. 


\section{Convención sobre los Derechos de la Niñez y Adolescencia}

La Convención sobre los Derechos de la niñez y adolescencia consagra tres principios básicos a los que deberá ajustarse la política nacional sobre niñez en Bolivia, a saber, Interés Superior de la Niñez y Adolescencia, Protección Integral y Garantía Jurídica, donde se comprende que el interés superior de la niñez y adolescencia es todo aquello que favorezca su desarrollo físico, psicológico, moral y social para lograr el pleno y armonioso desenvolvimiento de su personalidad por lo tanto este principio implica que en todo momento las políticas, acciones y toma de decisiones relacionadas con la niñez, deberán desarrollarse de forma que se procure el beneficio directo del niño o niña a quienes van dirigidas.

\section{Convención Internacional sobre Derechos del Niño y Adolescentes de 1989}

En este sentido es preciso determinar que el interés superior de la niñez y adolescencia, al que hace referencia la Convención, es un concepto que busca describir de manera general el "bienestar de los menores de edad", sin embargo, a raíz de que cada caso es especial y único, no existe una definición general, por ello debe ser evaluado de manera individual, teniendo en cuenta las características del caso. No obstante, si bien era necesario desde un comienzo tener claro el concepto de interés superior del menor, más adelante se ahondara en el mismo, ya que precisamente en él, se fundamentan las razones de evolución legislativa que nos llevarán a la consagración del Código de Niña, niño y adolescente boliviano.

\section{Derechos de las adolescentes}

Según Pacheco (2004):

Los derechos de los menores de edad, denominados Derecho del niño, niña y adolescente es el conjunto de normas que tienen por objeto reglar la actividad comunitaria en relación con la niñez y adolescencia, regulando sus derechos y deberes en el marco de la prevención, atención y protección de esta categoría social, buscando su desarrollo integral en función a su interés superior. Los derechos de la niñez y adolescencia, en resumen, se definen como el conjunto de derechos humanos que tienen como sujeto titular de los mismos al niño, es decir a aquella persona que está comprendida entre los cero y dieciocho años. (p.12)

Si bien se sabe los niños y adolescentes son el futuro del mundo, ellos son los llamados a cambiar la situación de vida para que se deje de un lado la violencia, desequilibrio, inequidad, injusticia, que vive toda la sociedad en conjunto y en especial las adolescentes en los centros de acogida. De ahí nace la importancia de analizar en qué condiciones viven las adolescentes en los centros de acogidas y que 
protección les brinda el Estado y las diferentes organizaciones que protegen a los menores de edad, para poder desarrollarse en un entorno favorable y crecer como personas de bien necesitan tener una vida digna sin maltratos, con tolerancia, amor, cariño, protección y todos los factores que un menor necesita simplemente por el hecho de ser un niño, niña o adolescente.

\section{Los centros de acogida de adolescentes}

El papel del Estado, con relación a lo institucional, como garante de los derechos, legitima el proceso de socialización de los actores sociales en beneficio de proporcionar mejores condiciones, para la satisfacción de necesidades que demanda la sociedad, producto de la aprehensión que ha sido generada por los cambios históricos y culturales, como lo ha sido el desempleo y la pobreza en las diferentes sociedades y que repercute en problemas sociales. En este contexto, se concluye que la familia es la más afectada.

Desde esta perspectiva, Fernández y Fuertes (2007), definen los centros de acogida como medida protectora a menores, mediante la cual se establecen elementos de una vida en colectividad, donde el menor y el adolescente se sienten apoyado y unido a sus iguales. Es el lugar donde aprenden a tomar decisiones en grupo, valores y patrones de conducta de interacción social, que no fueron desarrollados en el ambiente familiar.

\section{Centro de Acogida}

Se entiende por Centro de Acogida, a la institución dependiente económica y administrativamente de la Instancia Técnica Departamental de Política Social, que cumple una función de acogimiento y servicio integral con fines de protección a niños, niñas y adolescentes, en el marco de la Ley 548. (SEDEGES Potosi)

Adolescente. Persona comprendida entre los 12 a los 18 años de edad, sujeto de derechos y deberes (SEDEGES Potosí).

Interna o Acogida. La adolescente integrada a un Centro de Acogida mediante Orden Judicial emitido por del Juzgado Público de la Niñez y Adolescencia, como una medida de protección sin que esto implique la suspensión, pérdida o extinción de autoridad y por ende de las obligaciones de los padres biológicos, salvo casos expresamente señalados por autoridad competente. (SEDEGES Potosí).

\section{Equipo Técnico Multidisciplinario}

El mismo está conformado por profesionales en Trabajo Social, Psicología, Pedagogía, Psicopedagogía y Derecho cuando estos fueran asignados sus funciones estarán sujetas a las determinaciones contempladas en la Ley 548 y al manual de funciones de la entidad. 


\section{Trabajador social}

El trabajador Social actúa en el ámbito de las relaciones entre sujetos sociales y entre estos con el Estado. En el caso de la investigación se ocupa del bienestar de las adolescentes en el centro de acogida, desarrolla un conjunto de acciones de carácter socioeducativo, que inciden en la reproducción material y social de la vida de las adolescentes, en una perspectiva de transformaciones sociales.

\section{Psicólogo}

Es imprescindible la comunicación del psicólogo con la administración del centro, hacía preciso delimitar una serie de funciones específicas de profesional, a fin de optimizar su trabajo individual y coordinado, con la red multidisciplinar en el que dichas funciones se llevan a cabo. El enfoque del psicólogo, lejos de circunscribirse únicamente a la red de servicios sociales busca de una manera más amplia, mejorar la calidad de vida y el bienestar de las personas, superando el marco conceptual que incluye términos como marginación, inadaptación, entre otros, de esta manera pretende superar enfoques basados en las carencia y las patologías, para asumir un papel de la intervención en situaciones de crisis, como la prevención de las mismas, de cara a maximizar sus habilidades y competencias de análisis y transformación de su realidad social.

\section{Objetivos de los centros de acogidas}

Según Oliván (1994) la función de acogida:

Se entiende por tal, la función de recepción de un menor que ha sido maltratado o está en riesgo de serlo. Al ingreso es muy importante detectar indicadores físicos y emocionales relevantes que orienten el diagnóstico y ver su evolución (por ejemplo aspecto físico y nutricional, vestimenta e higiene, retrasos en el desarrollo psicomotor, alteraciones en las funciones del sueño, alimentación o control de esfínteres como tristeza, indiferencia, ansiedad, temor, extrañeza o no ante el desconocido) (p. 98).

Esta función de acogida, para conseguirse con éxito, requiere una planificación precisa y adaptable a cado caso, ya que suele comportar en el menor fenómenos traumáticos, como la posible urgencia en que se haya producido el ingreso, la traumática separación de sus familiares que pueda haber acaecido, el miedo a la novedad, la posible sensación de culpabilidad por haber sido descubierta su situación, el miedo a las represalias de sus maltratadores. Respecto a los criterios de ubicación del menor en el centro se tiende a mantener a una cierta homogeneidad en las edades y en los niveles de desarrollo. 


\section{Principales Instancias Involucradas con las Adolescentes}

\section{Las Defensorías de la Niñez y Adolescencia}

Entre las atribuciones más relevantes de las defensorías de la niñez y adolescencia relacionada con la protección de menores se tienen las siguientes:

- Presentar denuncia ante las autoridades competentes por infracciones o delitos cometidos en contra de los derechos de niños, niñas y adolescentes e intervenir en su defensa en las instancias administrativas o judiciales sin necesidad de mandato expreso.

- Derivar a la autoridad judicial los casos que no son de su competencia o han dejado de ser.

- Disponer las medidas de protección social a niños, niñas y adolescentes previstas por este cuerpo Legal.

\section{Instancia técnica de política social (EX SEDEGES)}

Dentro las atribuciones más relevantes del Ex SEDEGES está el cuidado y protección del Centro de Acogida 10 de noviembre donde se encuentran acogidas 24 adolescentes, sus atribuciones son las siguientes:

- Cumplir y hacer cumplir las políticas y normas establecidas en asuntos de género, generacionales, familia servicios sociales.

- Establecer prioridades departamentales con la relación a la situación de niños, niñas y adolescentes para su presentación a la Comisión de la Niñez y Adolescencia de la prefectura.

- Promover programas y acciones vinculadas a la problemática de los niños y niñas de las calles y defensa de sus derechos.

- Promover y velar por el respeto y vigencia de los derechos de la familia, la mujer, niño, niña adolescente y adulto /a mayor, su defensa socio-jurídica, así como prevenir situaciones actos atentatorios contra su integridad física, moral psicológica.

- Acreditar a las instituciones públicas y privadas de Servicio Social y de atención a niños, niñas y adolescentes, en el ámbito departamental, llevar un registro permanente y actualizado. 


\section{Marco jurídico sobre los Derechos de las Adolescentes en el Estado Plurinacional de Bolivia}

\section{Constitución Política del Estado}

La Constitución Política del Estado es el máximo instrumento de la legislación boliviana, dedica directamente a los menores de edad varias de sus disposiciones como ser los derechos fundamentales y fundamentalísimos, asimismo en la Sección V el cual hace referencia a los Derechos de la Niñez, Adolescencia en los siguientes artículos 13, 58, 59, 60, 61.

\section{El Código del Niño, Niña y Adolescente}

Esta normativa regula el ejercicio de los Derechos de la Niña, Niño y Adolescente; su finalidad es la protección y garantía de esos derechos para su desarrollo pleno e integral y exigir el cumplimiento de los mismos, detallados en los siguientes artículos: 1, 5,12,13, 15,17,18, 141, 142, 145,146, 147, 148, 159, 162, 168.

\section{Código Penal}

El Derecho Penal como ente regulador de la conducta del ser humano dentro de la sociedad también hace efectiva su aportación al Código del Niño, Niña y Adolescencia, con el objetivo de asegurar los valores elementales sobre los cuales descansa la convivencia humana pacífica mediante la aplicación de leyes penales por medio de una pena, una medida de seguridad o corrección.

\section{MÉTODO}

La investigación metodológicamente estuvo bajo el enfoque cuantitativo y cualitativo. El primero, trató de determinar la fuerza de asociación o correlación entre las dos variables fijas, el segundo, porque permitió conocer el fenómeno social relacionado con la doctrina correspondiente a los derechos de Las adolescentes en el centro como un problema social-jurídico que afecta a la sociedad en su conjunto enfocado a la búsqueda de repuestas jurídicas y posibles prácticas para solucionar el problema.

El tipo de Investigación fue Descriptivo, ya que se realizó un registro detallado del comportamiento específico de dos variables identificadas; la independiente (Reglamento Interno para el centro de acogida 10 de noviembre) y la dependiente (Incumplimiento de los derechos de Las adolescentes en el centro de acogida 10 de noviembre). A su vez, en la investigación el diseño fue documental de campo, dado a que los datos de interés fueron recogidos en forma directa de la realidad.

Por otro lado, algunos de los métodos usados para cumplir con el objetivo central de la investigación fueron: 
Análisis-Síntesis, con la finalidad de estructurar los componentes sobre el incumplimiento de los derechos de las adolescentes en el centro de acogida 10 de noviembre, se utilizó el estudio en la literatura consultada por todos los métodos y en la interpretación de los datos empíricos. Así como para arribar a conclusiones objetivas a partir de fuentes bibliográficas y sistematizar las características de la propuesta referida a una Propuesta de Reglamento Interno del Centro de Acogida 10 de noviembre.

Histórico-Lógico, se estudió los documentos históricos existentes como Bibliografías, Teorías, Derecho Comparado, Leyes y otras disposiciones legales referidas al incumplimiento de los derechos de Las adolescentes del centro de acogida 10 de noviembre.

A su vez, esta investigación usó la encuesta y entrevista como técnica, con el fin de recabar una información fidedigna aplicada a Las adolescentes y personal del centro de acogida 10 de noviembre, con la finalidad de recolectar datos certeros. $Y$ el instrumento fue el cuestionario. La población usada fue la perteneciente en el centro de acogida 10 de noviembre de la Ciudad de Potosí- Bolivia. La muestra usada fueron 24 adolescentes , 6 encargados del personal del Centro de acogida y las entrevistas fueron a 1 Juez del Juzgado Público de la Niñez y Adolescencia , 2 abogados de la Defensoría de la Niñez y Adolescencia, especialistas en la materia

RESULTADOS

Una vez, aplicado los instrumentos para el estudio se obtuvieron como resultado que en el centro de acogida 10 de noviembre de la Ciudad de Potosí-Bolivia, la atención de las adolescentes no practicaron ninguno de los valores como la comprensión, fraternidad, cooperación, entre otros, por no contar con equipos multidisciplinarios de profesionales adecuadamente capacitados con formación específica en disciplina sociales, culturales y recreativas. Además, de no respetar su dignidad, opiniones y forma de ser, por lo que tanto las adolescentes son vulnerables, y están expuestas a todo tipo de maltrato siendo dependientes del centro de acogida, necesitan el apoyo entre ellas, cuidadores y personal del centro, este apoyo no solo necesita para su supervivencia, sino también para el bienestar social y psicológico.

Además, se logró determinar que en el centro de acogida no responden adecuadamente a las necesidades e intereses de las adolescentes ya que no existe el personal adecuado y capacitado. También se evidenció que el centro de acogida 10 de noviembre no cuenta con un reglamento interno donde pueda regirse el funcionamiento, cuidados, alimentación, educación, vestimenta y demás factores que son primordiales para que las adolescentes puedan llevar una vida con un desarrollo pleno como cualquier otra adolescente. De tal modo, en dicho centro, las adolescentes no son sancionadas bajo una normativa, por no existir la misma, así que, el mismo 
personal se encarga de hacerlo de manera deliberada, perjudicando a las adolescentes a nivel psicológica y moral.

Así mismo, en la investigación se acudió a diferentes instancias verificándose que no existe un Reglamento interno con el que se maneje este centro de acogida 10 de noviembre, motivo por el cual funciona a conveniencia y criterio de funcionarios y personal de esta institución.

Es por ello, que se requirió de un Reglamento Interno que regule adecuadamente las funciones del personal, personal que tiene que ser especializado y como se debe actuar en las distintas situaciones, pero también se precisa que la Gobernación asigne más recursos económicos no solo para aumentar el personal y capacitarla sino también para que el material, infraestructura, entre otros, sean previstos oportunamente y estén en condiciones adecuadas, buscando siempre el interés superior de las adolescentes

\section{Reglamento Interno para el cumplimiento de los derechos de las adolescentes del centro de acogida 10 de noviembre de la Ciudad de Potosí-Bolivia}

En este sentido, cumpliendo con el objetivo principal de la investigación se elaboró un reglamento interno para el cumplimiento de los derechos de las adolescentes del centro de acogida 10 de noviembre de la Ciudad de Potosí-Bolivia para velar por el cumplimiento de los derechos de las adolescentes dentro del centro.

\section{Fundamentación Jurídica}

Para desarrollar la fundamentación jurídica, se tomó en cuenta disposiciones legales como la Constitución Política del Estado y la Ley N548 Nuevo Código Niña, Niño y Adolescente que hace referencia a la investigación.

En la nueva Constitución Política del Estado se tomaron los artículos 59 y 60, donde hace referencia a que toda niña, niño y adolescente tiene derecho a su desarrollo integral. Y Estado, sociedad, y familia lo deben garantizar

En la Ley $\mathrm{N}^{\circ} 548$ se tomaron cuatro artículos donde se determina las garantías, principios, prioridad, entre otros aspectos (artículos 54, 55, 56, 173 y 174)

Así que, el reglamento se conformó de la siguiente manera:

Título I. Marco de referencia del centro de acogida 10 de noviembre. Capítulo I, aspectos generales, como lo son las definiciones de centro de acogida, adolescentes, interna; además, el objetivo general del reglamento, propósito, ámbito de aplicación, y naturaleza.

Título II. Del contenido del centro de acogida 10 de noviembre. Capítulo I, Prestaciones sociales como lo son, la atención social, alimentación, higiene, ropería, lavandería y atención sanitaria. Capitulo II, Derechos y deberes de las Internas del centro. 
Título III. De la organización y funcionamiento del Centro de Acogida 10 de Noviembre. Capítulo I, Unidades organizativas como la dirección, personal, del equipo técnico multidisciplinario, de las funciones del personal administrativo, prohibición y faltas de personal, sanciones al personal, régimen de salidas, régimen de ausencias, régimen de visitas, del acogimiento del centro, cuadernos de registro. Capítulo II, régimen disciplinario, faltas leves, faltas graves, faltas muy graves, sanciones,

Título IV. De la participación de las personas usuarias del servicio. Capítulo I, Mecanismo de participación,

\section{CONCLUSIONES}

Cumpliendo con el objetivo principal del artículo se llegó a las siguientes conclusiones:

Se pudo establecer, que si bien se sabe que los derechos de las adolescentes están protegidos y garantizados por normativa jurídica nacional e internacional, se observa que el incumplimiento de sus derechos sigue dando de qué hablar, dejando de lado su dignidad como persona humana.

De acuerdo a los resultados obtenidos se evidenció que las adolescentes sufren incumplimiento de sus derechos, llegando hacer un análisis se encuentra diferentes factores que intervienen en este maltrato, como los fueron no tener estrategias claras y bien definidas por parte de instancias municipales, manteniendo la lógica de que muchos de los derechos de las adolescentes y sobre todo temas referidos a la protección y cuidado no son respetados, hecho que tiene un impacto significativo en el desarrollo social y humano de la población.

Para finalizar, se sustentó que el Estado a través del Gobierno Autónomo Municipal de Potosí, debe aprobar políticas dirigidas al sector de los centros de acogida especialmente a una reglamentación para el buen funcionamiento del mismo, que deben garantizar a las adolescentes sus derechos, por lo que la presente Reglamentación presenta y detalla todo lo necesario para que el centro pueda llevar un buen funcionamiento respetando y cumpliendo los derechos de las adolescentes.

\section{REFERENCIAS}

Alcántara, M. V. (2010). Víctimas invisibles: los hijos de las mujeres víctimas de la violencia de género. Tesis doctoral Universidad de Murcia

Cárdenas, B. (2012). Percepción de los trabajadores sociales, educadores y psicológicos sobre los centros de acogida: caso España y Alemania. Tesis Doctorales. Universidad de Alicante

Constitución Política del Estado de Bolivia. (2009). Derechos de la Niñez, Adolescencia Sección V, artículos 13, 58, 59, 60, 61
Derechos Humanos, D. U. (1948). Declaración Universal de los Derechos humanos. Asamblea General de las Naciones Unidas, 10

Fernández, D., y Fuertes, Z. (2007). El acogimiento residencial en la protección a la infancia. Madrid: Pirámide

Ley No 548 Código Niña, Niño y Adolescente (2014). Ministerio de Justicia. La Paz, Bolivia

Morlachetti, A. (2014). La Convención sobre los Derechos del Niño y la protección de la infancia en la normativa internacional de 
derechos humanos. Derechos Humanos de los grupos vulnerables Manual, 21

Oliván. G, (1994). Centros de Acogida de Menores. Anales españoles de pediatría, p. 98
Pacheco, S. (2004). Derechos de la Niñez y Adolescencia, teoría y práctica- Editorial Alexander, Cochabamba-Bolivia

Servicio Departamental de Gestión Social Potosí Bolivia 